\title{
From Dinuclear Systems to Close Binary Stars: Application to Source of Energy in the Universe
}

\author{
V.V. Sargsyan ${ }^{1,2}$, H. Lenske ${ }^{2}$, G.G. Adamian ${ }^{1}$, and N.V. Antonenko ${ }^{1}$ \\ ${ }^{1}$ Joint Institute for Nuclear Research, 141980 Dubna, Russia, \\ ${ }^{2}$ Institut für Theoretische Physik der \\ Justus-Liebig-Universität, D-35392 Giessen, Germany
}

(Dated: January 1, 2019)

\begin{abstract}
The evolution of close binary stars in mass asymmetry (transfer) coordinate is considered. The conditions for the formation of stable symmetric binary stars are analyzed. The role of symmetrization of asymmetric binary star in the transformation of potential energy into internal energy of star and the release of a large amount of energy is revealed.

PACS numbers: 26.90.+n, 95.30.-k
\end{abstract}

Keywords: close binary stars, mass transfer, mass asymmetry 


\section{INTRODUCTION}

Because mass transfer is an important observable for close binary systems in which the two stars are nearly in contact [1 1 ] $]$, it is meaningful and necessary to study the evolution of these stellar systems in the mass asymmetry coordinate $\eta=\left(M_{1}-M_{2}\right) /\left(M_{1}+M_{2}\right)$ where $M_{i},(i=1,2)$, are the stellar masses. In our previous work [9], we used classical Newtonian mechanics and study the evolution of the close binary stars in their center-ofmass frame by analyzing the total potential energy $U(\eta)$ as a function of $\eta$ at fixed total mass $M=M_{1}+M_{2}$ and orbital angular momentum $L=L_{i}$ of the system. The limits for the formation and evolution of the di-star systems were derived and analyzed. We used theoretical methods which are being applied successfully to corresponding processes in nuclear systems where mass asymmetry plays an important role as the collective coordinate governing fusion of two heavy nuclei [10,11]. Nuclear dynamics, of course, is quite different from the gravitational interactions in di-stars. Nuclear reactions are dominated by shortranged strong interactions, to which minor contributions of long-range (repulsive) Coulomb and centrifugal forces are superimposed. However, extending the methods and results from the femto-scale of microscopic nuclear physics to macroscopic binary stellar systems, we obtained that the driving potentials $U(\eta)$ for the di-star systems looks like the driving potentials for the microscopic dinuclear systems [9-11].

One should stress that in Ref. [9] the mass-asymmetry-independent structural factor was used as a free parameter. In the general, the dimensionless structural factor $\omega_{i}$ is determined by the density profile of the star. In the present paper, we generalize the approach of Ref. 9] by employing the values of the structural factor of the stars from the model of Ref. [7], Note that the model of Ref. 17] well describes the observable temperature-radius-massluminosity relations of stars, especially binary stars, the spectra of seismic oscillations of the Sun, distribution of stars on their masses, magnetic fields of stars and etc. The stellar radii, masses and temperatures are expressed by the corresponding ratios of the fundamental constants, and individuality of stars are determined by two parameters - by the charge and mass numbers of nuclei, from which a star is composed [7].

Our theoretical approach is introduced in Sect. [II. We explore the landscape of the total potential energy of the close binary system, searching specifically for the evolution paths in the mass asymmetry coordinate. In Sect. III the theoretical method is applied to concrete 
close binaries. It is shown that the mass transfer is one of the important sources of the transformation of the gravitational energy to other types of energy in the universe. In Sect. IV the obtained results are summarized.

\section{THEORETICAL METHOD}

The differential of total energy of di-star system as a function of relative distance $\mathbf{R}$ of two stars, conjugate canonical momentum $\mathbf{P}$ and mass asymmetry coordinate $\eta$ reads as

$$
d E(\mathbf{R}, \mathbf{P}, \eta)=\frac{\partial E}{\partial t} d t+\frac{\partial E}{\partial \mathbf{R}} d \mathbf{R}+\frac{\partial E}{\partial \mathbf{P}} d \mathbf{P}+\frac{\partial E}{\partial \eta} d \eta
$$

The kinetic energy in $\eta$ is assumed to be small and can be disregarded. In the center of mass system, the total energy of the di-star system is a sum of radial and orbital part of kinetic energies, and the potential energy. For the reasons seen below, we attach the orbital kinetic energy part to the star-star interaction $V$. In this case the expression of the total energy of the di-star system reads as

$$
E=\frac{P_{R}^{2}}{2 \mu}+U
$$

where $P_{R}$ is the radial component of the momentum $\mathbf{P}$ and $\mu=\mu(\eta)=\frac{M_{1} M_{2}}{M}=\frac{M}{4}\left(1-\eta^{2}\right)$ is the reduced mass. The total potential energy of the di-star system

$$
U=U_{1}+U_{2}+V
$$

is given by the sum of the potential energies $U_{i}(i=1,2)$ of the two stars and star-star interaction potential $V$. The radiation energy is neglected because the absolute values of the gravitational energy and the intrinsic kinetic energy are much larger than the radiation energy. The energy of the star " $i$ " is

$$
U_{i}=-\omega_{i} \frac{G M_{i}^{2}}{2 R_{i}}
$$

where $G, M_{i}$, and $R_{i}$ are the gravitational constant, mass, and radius of the star, respectively. Employing the values of the dimensionless structural factor

$$
\omega_{i}=1.644\left(\frac{M_{\odot}}{M_{i}}\right)^{1 / 4}
$$


and radius

$$
R_{i}=R_{\odot}\left(\frac{M_{i}}{M_{\odot}}\right)^{2 / 3}
$$

of the star from the model of Ref. [7], we obtain

$$
\begin{aligned}
U_{i} & =-\omega_{0} G M_{i}^{13 / 12} / 2, \\
\omega_{0} & =1.644 \frac{M_{\odot}^{11 / 12}}{R_{\odot}},
\end{aligned}
$$

where, $M_{\odot}$, and $R_{\odot}$ are mass and radius of the Sun, respectively. Because the average density $\rho_{i}=M_{\odot} \rho_{\odot} / M_{i}$ ( $\rho_{\odot}$ is the average density of the Sun) increase with decreasing the mass $M_{i}$ of star [7], the structural factor $\omega_{i}$ depends on $M_{i}$ in Eq. (5). The change of $\eta$ from 0 to 1 leads to the change of $\omega_{1}$ by about of $16 \%$. Note that in our work [9] the structural factor $\omega_{i}$ was used as a free mass-asymmetry-independent parameter.

Because the two stars rotate with respect to each other around the common center of mass, the star-star interaction potential contains, together with the gravitational energy $Q$ of the interaction of two stars, the kinetic energy of orbital rotation $V_{\text {rot }}$ :

$$
V(R)=Q+V_{\text {rot }}=-\frac{G M_{1} M_{2}}{R}+\frac{\mu v^{2}}{2},
$$

where $v=\left(G M\left[2 / R-1 / R_{m}\right]\right)^{1 / 2}$ and $R_{m}$ are the speed and the semimajor axis of the elliptical relative orbit, respectively $[1,5,7,8,8]$. Finally, one can derive the simple expression for the star-star interaction potential

$$
V\left(R_{m}\right)=-\frac{G M_{1} M_{2}}{2 R_{m}} .
$$

Because of Kepler's laws

$$
R_{m}=\left(\frac{\mu_{i}}{\mu}\right)^{2} R_{m, i}
$$

where the index "i" denotes the initial value of reduced mass and distance between the initial (before transfer) binary star, Eq. (8) is rewritten as

$$
V\left(R_{m}\right)=-\omega_{V} G\left(M_{1} M_{2}\right)^{3} / 2,
$$

where

$$
\omega_{V}=\frac{1}{M^{2} \mu_{i}^{2} R_{m, i}} .
$$


The final expression for the total potential energy (3) of the di-star system is

$$
U=-\frac{G}{2}\left(\omega_{0}\left[M_{1}^{13 / 12}+M_{2}^{13 / 12}\right]+\omega_{V}\left[M_{1} M_{2}\right]^{3}\right)
$$

Using the mass asymmetry coordinate $\eta$ instead of masses $M_{1}=\frac{M}{2}(1+\eta)$ and $M_{2}=\frac{M}{2}(1-\eta)$, we rewrite Eq. (11):

$$
U=-\frac{G M_{\odot}^{2}}{2 R_{\odot}}\left(\alpha\left[(1+\eta)^{13 / 12}+(1-\eta)^{13 / 12}\right]+\beta\left[1-\eta^{2}\right]^{3}\right)
$$

where

$$
\alpha=1.644\left(\frac{M}{2 M_{\odot}}\right)^{13 / 12}
$$

and

$$
\beta=\left(\frac{\pi^{2} M_{\odot}^{5} R_{\odot}^{3}}{32 G \mu_{i}^{6} P_{\text {orb }, i}^{2}}\right)^{1 / 3}\left(\frac{M}{2 M_{\odot}}\right)^{11 / 3} .
$$

To obtain $\beta$, we use the Kepler's third law connecting the semimajor axis

$$
R_{m, i}=\left(\frac{G M P_{o r b, i}^{2}}{4 \pi^{2}}\right)^{1 / 3}
$$

with the period $P_{o r b, i}$ of orbital rotation of the initial di-star system (orbital rotation of one star relative to the other). As seen from Eq. (12), the stability of the binary star system depends on the period $P_{\text {orb }, i}$ or the orbital angular momentum (with the mass asymmetry $\left.\eta_{i}\right)$ and the total mass $M$.

Employing Eq. (12), we can study the evolution of the di-star system in the mass asymmetry coordinate $\eta$. The extremal points of the potential energy as a function of $\eta$ are found by solving numerically the equation

$$
\frac{\partial U}{\partial \eta}=-\frac{G M_{\odot}^{2}}{2 R_{\odot}}\left(\frac{13}{12} \alpha\left[(1+\eta)^{1 / 12}-(1-\eta)^{1 / 12}\right]-6 \beta \eta\left[1-\eta^{2}\right]^{2}\right) .
$$

As seen, Eq. (14) is solved for $\eta=\eta_{m}=0$. At this value the potential has an extremum which is a minimum if

$$
\alpha<\frac{432}{13} \beta
$$

or

$$
P_{\text {orb }, i}<\frac{128.5 \pi}{\left(1-\eta_{i}^{2}\right)^{3}}\left(\frac{R_{\odot}^{3}}{G M_{\odot}}\right)^{1 / 2}\left(\frac{M}{2 M_{\odot}}\right)^{7 / 8}
$$

and a maximum if

$$
\alpha>\frac{432}{13} \beta
$$


The transition point is

$$
\alpha_{c r}=\alpha=\frac{432}{13} \beta=\frac{432}{13}\left(\frac{\pi^{2} M_{\odot}^{5} R_{\odot}^{3}}{32 G \mu_{i}^{6} P_{o r b, i}^{2}}\right)^{1 / 3}\left(\frac{M}{2 M_{\odot}}\right)^{11 / 3} .
$$

If there is a minimum at $\eta=0\left(\alpha<\alpha_{c r}\right)$, it is engulfed symmetrically by two barriers. Expanding Eq. (14) up to the third order in $\eta$ and solving it, we obtain the position of these barriers at $\eta= \pm \eta_{b}$, where

$$
\eta_{b}=2^{-1 / 2}\left(\frac{864^{2} \beta-22464 \alpha}{864^{2} \beta+3289 \alpha}\right)^{1 / 2} .
$$

So, at $\alpha<\alpha_{c r}$ the potential energy as a function of $\eta$ has two symmetric maxima at $\eta= \pm \eta_{b}$ and the minimum at $\eta=\eta_{m}=0$. The fusion of two stars with $\left|\eta_{i}\right|<\eta_{b}$ can occur only by overcoming the barrier at $\eta=+\eta_{b}$ or $\eta=-\eta_{b}$. With decreasing ratio $\alpha / \beta$, $B_{\eta}=U\left(\eta_{b}\right)-U\left(\eta_{i}\right)$ increases and the symmetric di-star system becomes more stable. The evolution of two stars with $\left|\eta_{i}\right| \neq 0$ and $\left|\eta_{i}\right|<\eta_{b}$ to the symmetric di-star configuration is energetically favorable. Hence, an initially asymmetric binary system $\left(|\eta|=\left|\eta_{i}\right|<\eta_{b}\right)$ is driven to mass symmetry, implying a flow of mass towards equilibrium and increase of the internal energy of stars by the amount $\Delta U=U\left(\eta_{i}\right)-U(\eta=0)$ (Fig. 1a). At $\alpha \geq \alpha_{c r}$, $\eta_{m}=\eta_{b}=0$ and we have the inverse $U$-type potential with maximum at $\eta=0$. In such a system, the fusion of stars (one star "swallows" the other star) is the only mode of evolution in $\eta$ transforming in the end the di-star into a mono-star with the release of energy $E_{f}=U\left(\eta_{i}\right)-U(\eta=1)$ (Fig. 1b).

As seen, $\eta_{b} \rightarrow 2^{-1 / 2} \approx 0.71$ if $\beta \gg \frac{1}{66} \alpha$. In this case the condition $0<\eta_{b}<2^{-1 / 2}$ means that in the asymmetric system with mass ratio $M_{1} / M_{2}>\left(1+2^{1 / 2}\right)^{2} \approx 6$ the stars fuse. Thus, di-stars with $|\eta|>\eta_{b}$ are unlikely to exist for sufficiently long time. Indeed, the binary stars with a large mass ratio are very rare objects in the universe.

\section{APPLICATION TO CLOSE BINARIES}

In the calculations, we assume that the orbital angular momentum $L_{i}$ and the total mass $M$ are conserved during the conservative evolution of the di-star in mass asymmetry coordinate $\eta$. The orbital angular momentum $L_{i}$ is calculated by using the experimental masses $M_{i}$ of stars and period $P_{o r b, i}$ of their orbital rotation (see Appendix A). 
One can express the potential energy (12) in units of

$$
u=u_{1}+u_{2}+x v=(1+\eta)^{13 / 12}+(1-\eta)^{13 / 12}+x\left(1-\eta^{2}\right)^{3},
$$

where $x=\beta / \alpha=\frac{G M_{\odot}^{3} R_{\odot}}{3.288 L_{i}^{2}}\left(\frac{M}{2 M_{\odot}}\right)^{47 / 12}$. As seen from Fig. 2, at relatively large $x>0.025$ the $u$ as a function of $\eta$ has two asymmetric maxima and the minimum at symmetry. The decrease of $x$ leads to the change of the shape of potential energy in $\eta: \eta_{b}$ approaches $\eta_{m}=0$ and the height of barrier $B_{\eta}$ in $\eta$ decreases. At $x=0.025$, we have the inverse $U$-type symmetric potential with maximum at $\eta=0$, and the di-star system becomes unstable with respect to the matter transfer coordinate. The asymmetrization of system is energetically favorable. The parameter $x \sim M^{47 / 12} / L_{i}^{2}$ depends on $M$ and $L_{i}$. For example, one can decrease $x$ with increasing $L_{i}$ or decreasing $M$.

Various di-stars have different $M$ and $L_{i}$ and, correspondingly, the potential energy shapes. The potential energies (driving potentials) $U(\eta)$ of the close di-star systems versus $\eta$ are presented in Figs. 3-7. For all systems shown, $\alpha<\alpha_{c r}$ or

$$
L_{i}<\left[10.1 G R_{\odot} M_{\odot}^{3}\right]^{1 / 2}\left(\frac{M}{2 M_{\odot}}\right)^{47 / 24}
$$

respectively the potential energies have symmetric barriers at $\eta= \pm \eta_{b}$ and the minimum at $\eta=\eta_{m}=0$. As seen in Fig. 3, the barrier in $\eta$ appears as a result of the interplay between the total gravitational energy $U_{1}+U_{2}$ of the stars and the star-star interaction potential $V$. Both energies have different behavior as a function of mass asymmetry: $U_{1}+U_{2}$ decreases and $V$ increases with changing $\eta$ from $\eta=0$ to $\eta= \pm 1$. One should stress that the driving potentials $U(\eta)$ for the di-star systems looks like the driving potentials for the microscopic dinuclear systems [10, 11]. Note that the same conclusion was derived in Ref. [9] by using the mass-asymmetry-independent (constant) structural factors $\omega_{i}$.

The evolution of the di-star system depends on the initial mass asymmetry $\eta=\eta_{i}$ at its formation. If the original di-star is asymmetric, but $\left|\eta_{i}\right|<\eta_{b}$, then it is energetically favorable to evolve in $\eta$ to a configuration in the global minimum at $\eta=0$, that is, to form a symmetric di-star system. The matter of a heavy star can move to an adjacent light star enforcing the symmetrization of di-star without additional driving energy. The symmetrization of asymmetric binary star leads to the decrease of potential energy $U$ or the transformation of the potential energy into internal energy of stars. The resulting symmetric di-star is created at large excitation energy. For example, for the binary systems 
RR Cen $\left(\eta_{i}=0.65\right)$, V402 Aur $\left(\eta_{i}=0.66\right)$, and V921 Her $\left(\eta_{i}=0.61\right)$, the internal energies of stars increase during symmetrization by amount $\Delta U=U\left(\eta_{i}\right)-U(\eta=0)=2 \times 10^{41}, 10^{41}$, and $10^{41} \mathrm{~J}$, respectively. Because the most of close binary stars are asymmetric ones, the symmetrization process leads to the release of a large amount of energy in these systems and can be an important source of energy in the universe (see Tables I, II, and III). Note that accounting for the loss of angular momentum will lead to an increase of the value $\Delta U$.

If $\left|\eta_{i}\right|>\eta_{b}$ or $\eta_{b}=0$, the di-star system is unstable and evolves towards the mono-star system, thus, enforcing the asymmetrization of the di-star. The matter is transferred from the light star to the heavy star even without additional external energy. We found only one close binary system $\alpha \mathrm{Cr} \mathrm{B}\left(M_{1}=2.58 M_{\odot}, M_{2}=0.92 M_{\odot}, \omega_{1}=1.30, \omega_{2}=1.68\right.$, $\beta / \alpha=0.039$ ), for which $\left|\eta_{i}\right|=0.47>\eta_{b}=0.33$ (Fig. 3).

Because the fusion barriers $B_{\eta}$ in $\eta$ are quite large for the systems with $\left|\eta_{i}\right|<\eta_{b}$ in Tables I, II, and III, the formation of a mono-star from the di-star system by the thermal diffusion in mass asymmetry coordinate is strongly suppressed. Perhaps, the existence of barrier in $\eta$ is the reason why very asymmetric close double-stars systems with $\left|\eta_{i}\right|>\eta_{b}$ are rarely observed. This imposes restrictions on the asymmetric configurations with $|\eta|>\eta_{b}$ of the di-star systems. There can not be a stable di-star with a very light star, with only a fraction of the mass of the heavy companion.

The value of $\alpha$ becomes larger than $\alpha_{c r}$ and the minimum in $U(\eta)$ disappears, and di-star asymmetrization (fusion in $\eta$ coordinate) occurs as the result of a release of matter from one of the stars or an increase of orbital momentum due to the strong external perturbation, i.e. by the third object, or the spin-orbital coupling in the di-star. Because the close binary stars BW Aqr $\left(\eta_{i}=0.04, \eta_{b}=0.34\right)$, V $889 \mathrm{Aql}\left(\eta_{i}=0.04, \eta_{b}=0.30\right)$, V $541 \mathrm{Cyg}\left(\eta_{i}=0.02\right.$, $\left.\eta_{b}=0.19\right), \mathrm{V} 1143 \mathrm{Cyg}\left(\eta_{i}=0.02, \eta_{b}=0.27\right)$, and IT Cas $\left(\eta_{i}=0, \eta_{b}=0.49\right)$ have the smallest fusion barriers $B_{\eta}$ (Table I, Figs. 4 and 5), these systems may be a good candidates for such kind processes.

A spectacular recent case is KIC 9832227 which was predicted [13] to be merge in 2022, enlightening the sky as a red nova. For the fate of $\operatorname{KIC} 9832227\left(\eta_{i}=0.63, \eta_{b}=0.84\right)$, we predict that a fast merger is excluded (see Fig. 7). This di-star is driven instead towards the mass symmetry. The mass is transferring from heavy star to light one and the relative distance between two stars and the period of the orbital rotation are decreasing. A huge amount of energy $\Delta U \approx 10^{41} \mathrm{~J}$ is released during the symmetrization. As seen in Fig. 7, 
the di-stars KIC 9832227 and RR Cen $\left(\eta_{i}=0.65, \eta_{b}=0.85\right)$ have almost the same $\eta_{i}, \eta_{b}$, and potential energy shapes. So, the observation of the RR Cen di-star is also desirable. It should be stressed that the observational data of Ref. [14] negate the 2022 red nova merger prediction [13].

\section{SUMMARY}

The isolated close binary star systems evolve along well defined trajectories in classical phase space. We have shown that energy conservation is enough to fix the trajectory of the system in the potential energy landscape defined by the total mass and orbital angular momentum of system. Exploiting the stationarity of the total energy, stability conditions were derived and investigated as functions of the mass asymmetry parameter $\eta$. We have shown that this collective degree of freedom plays a comparable important role in macroscopic object as well as in microscopic dinuclear systems. In close di-star systems, the mass asymmetry coordinate can govern the fusion (merger) and symmetrization (due to the mass transfer) processes of two stars. An interesting aspect is that once $\eta$ has been determined e.g. by observation, it allows to conclude on the stellar structure parameters $\alpha$ or, likewise, $\omega_{i}$.

Our novel theoretical treatment of di-star system is based on the fact that after the formation process the lifetime of a di-star system is long enough to reach equilibrium conditions in the mass asymmetry coordinate. Hence, we could conclude that the system will be a member of the sample of all di-star and mono-star configurations with the probabilities depending on the potential energies of a given configuration. For all systems considered, $\alpha<\alpha_{c r}$ and the potential energies have symmetric barriers at $\eta= \pm \eta_{b}$ and the minimum at $\eta=\eta_{m}=0$. At $\alpha<\alpha_{c r}$, two distinct evolution scenario arise. Let the di-star system be initially formed with $\eta=\eta_{i}$. The two stars start to exchange matter where the fate of the binary depends critically on the mass ratio: If $\left|\eta_{i}\right|<\eta_{b}$ the system is driven to the symmetric di-star configuration (towards a global minimum of the potential landscape). However, if $\left|\eta_{i}\right|>\eta_{b}$ the system evolves towards the mono-star system. All asymmetric close binary stars considered, except $\alpha \mathrm{Cr}$ B, satisfy the condition $\left|\eta_{i}\right|<\eta_{b}$ and in these systems the symmetrization process occurs. Note that for many systems $U(\eta=0)<U(|\eta|=1$.

In the case of $\left|\eta_{i}\right|<\eta_{b}\left(\alpha<\alpha_{c r}\right)$, the symmetrization of stars leads to the release of 
a large amount of energy about $10^{41} \mathrm{~J}$ as radiation outburst and matter release. Thus, the symmetrization of stars in close binary systems due to the mass transfer is one of the important sources of the transformation of the gravitational energy to other types of energy in the universe. The symmetrization of binary system will lead to $M_{1} / M_{2} \rightarrow 1, T_{1} / T_{2} \rightarrow 1$ ( $T_{i}$ are the temperatures of stars), $L_{1} / L_{2} \rightarrow 1$ ( $L_{i}$ are the luminosities of stars), $R_{1} / R_{2} \rightarrow 1$ which are observable quantities.

\section{ACKNOWLEDGEMENTS}

V.V.S. acknowledge the partial supports from the Alexander von Humboldt-Stiftung (Bonn). This work was partially supported by Russian Foundation for Basic Research (Moscow) and DFG (Bonn). 


\section{Appendix A: The star-star interaction potential}

Because the two stars rotate with respect to each other around the common center of mass, the star-star interaction potential contains, together with the gravitational energy of interaction of two stars, the kinetic energy of orbital rotation:

$$
V(R)=-\frac{G M_{1} M_{2}}{R}+\frac{L^{2}}{2 \mu R^{2}},
$$

where $L$ is the orbital angular momentum of the di-star which is conserved during the conservative mass transfer. From the conditions $\partial V /\left.\partial R\right|_{R=R_{m}}=0$ and $\partial^{2} V /\left.\partial R^{2}\right|_{R=R_{m}}=$

$G \mu M / R_{m}^{3}>0$, we find the relative equilibrium distance between two stars corresponding to the minimum of $V$ :

$$
R_{m}=\frac{L^{2}}{G \mu^{2} M}
$$

Using Eq. (A2) and

$$
P_{\text {orb }}=2 \pi\left[R_{m}^{3} /(G M)\right]^{1 / 2}
$$

for the period of orbital rotation of the di-star system (Kepler's third law), we obtain

$$
L=L_{i}=\frac{2 \pi \mu R_{m}^{2}}{P_{\text {orb }}}=\mu\left(G M R_{m}\right)^{1 / 2}
$$

and derive the simple expression

$$
V\left(R_{m}\right)=-\frac{G M_{1} M_{2}}{2 R_{m}}=-\beta G\left(1-\eta^{2}\right)^{3} / 2
$$

where

$$
\beta=\frac{G M^{5}}{64 L_{i}^{2}}=\frac{G M_{\odot}^{5}}{2 L_{i}^{2}}\left(\frac{M}{2 M_{\odot}}\right)^{5}
$$

coincides with $\beta$ from Sect. 2 because

$$
L_{i}^{2}=\mu_{i}^{2} G M R_{m, i}=\mu_{i}^{2}\left(\frac{G^{4} M^{4} P_{o r b, i}^{2}}{4 \pi^{2}}\right)^{1 / 3} .
$$

Here, the relation $L=L_{i}$ follows from Eqs. (9) and (A2).

[1] Z. Kopal, Close binary systems (Shapman and Hall LTD, London, 1978).

[2] S.N. Shore, M. Livio, and E.P.J. van den Heuvel, Interacting binaries (Springer-Verlag, BerlinBudapest, 1994). 
[3] R.W. Hilditch, An introduction to close binary stars (Cambridge Univ. Press, Cambridge, 2001).

[4] A.A. Boyarchuk et al., Mass Transfer in close binary stars (Teylor and Francis, London, New York, 2002).

[5] P.P. Eggleton, Evolutionary processes in binary and multiple stars ( Cambridge Univ. Press, Cambridge, 2006).

[6] K.F. Khaliullin, Dissertation (Russian) (Sternberg Astronomical Institute, Moscow, 2004).

[7] B.V. Vasiliev, Astrophysics and astronomical measurement data (Fizmatlit, Moscow, 2012); Univ. J. Phys. Applic. 2, 257 (2014); 2, 284 (2014); 2, 328 (2014); http://astro07.narod.ru.

[8] A.M. Cherepashchuk, Close binary stars (Fizmatlit, Moscow, 2013), vol. I and II.

[9] V.V. Sargsyan, H. Lenske, G.G. Adamian, N.V. Antonenko, Int. J. Mod. Phys. E 45, 1850063 (2018).

[10] G.G. Adamian, N.V. Antonenko, and W. Scheid, Lect. Notes Phys. 848, Clusters in Nuclei Vol. 2, Ed. by Christian Beck (Springer-Verlag, Berlin, 2012) p. 165.

[11] G.G. Adamian, N.V. Antonenko, and A.S. Zubov, Phys. Part. Nucl. 45, 848 (2014).

[12] K. Yakut and P.P. Eggleton, Astrophys. J. 629, 1055 (2005); K. Gazeas and K. Stȩpień, MNRAS 390, 1577 (2008).

[13] L.A. Molnar, D.M. Van Noord, K. Kinemuchi, J.P. Smolinski, C.E. Alexander, E.M. Cook, B. Jang, H.A. Kobulnicky, C.J. Spedden, and S.D. Steenwyk, S.D. arXiv:1704.05502 (2017).

[14] Q.J. Socia et al., ApJL 864, L32 (2018). 

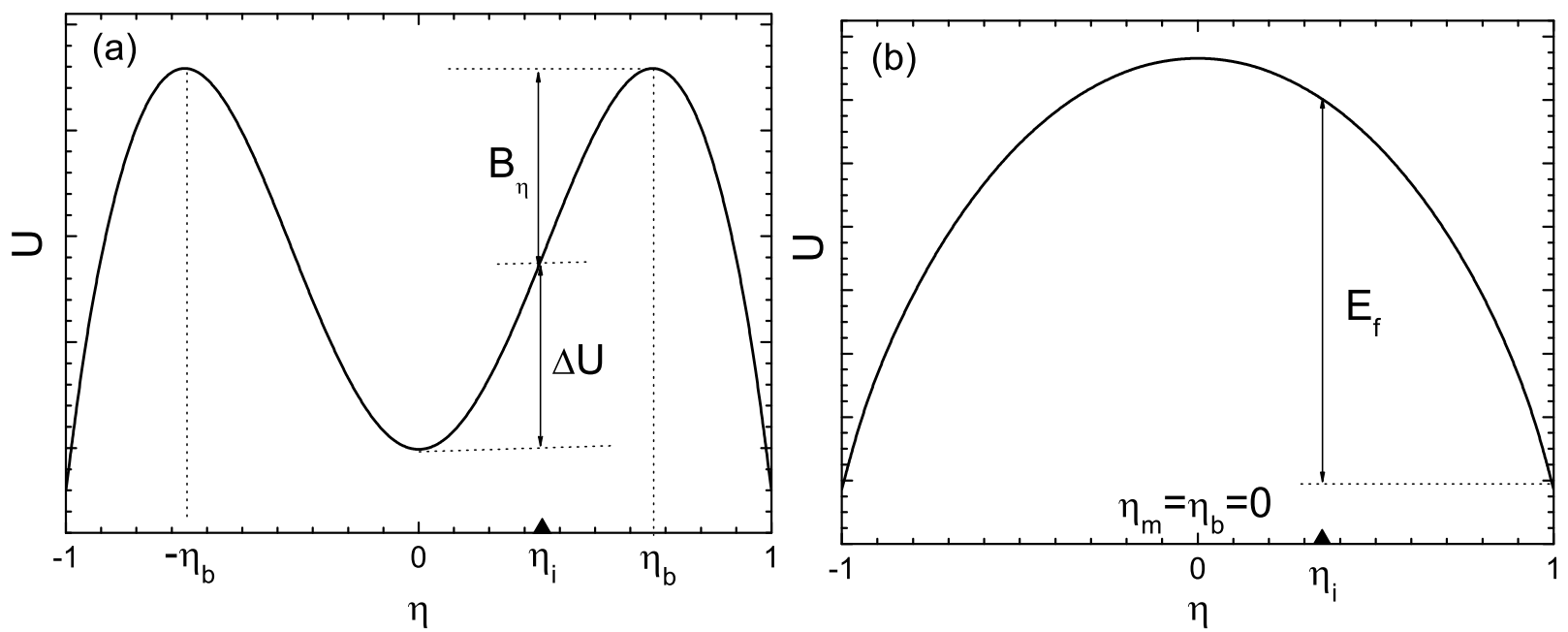

FIG. 1: The schematical drawings of the driving potential energy of the star-star system at $\alpha<\alpha_{c r}$ (a), and $\alpha>\alpha_{c r}$ (b). The arrows on $x$-axis show the corresponding initial binary stars. The notations used in the text are indicated.

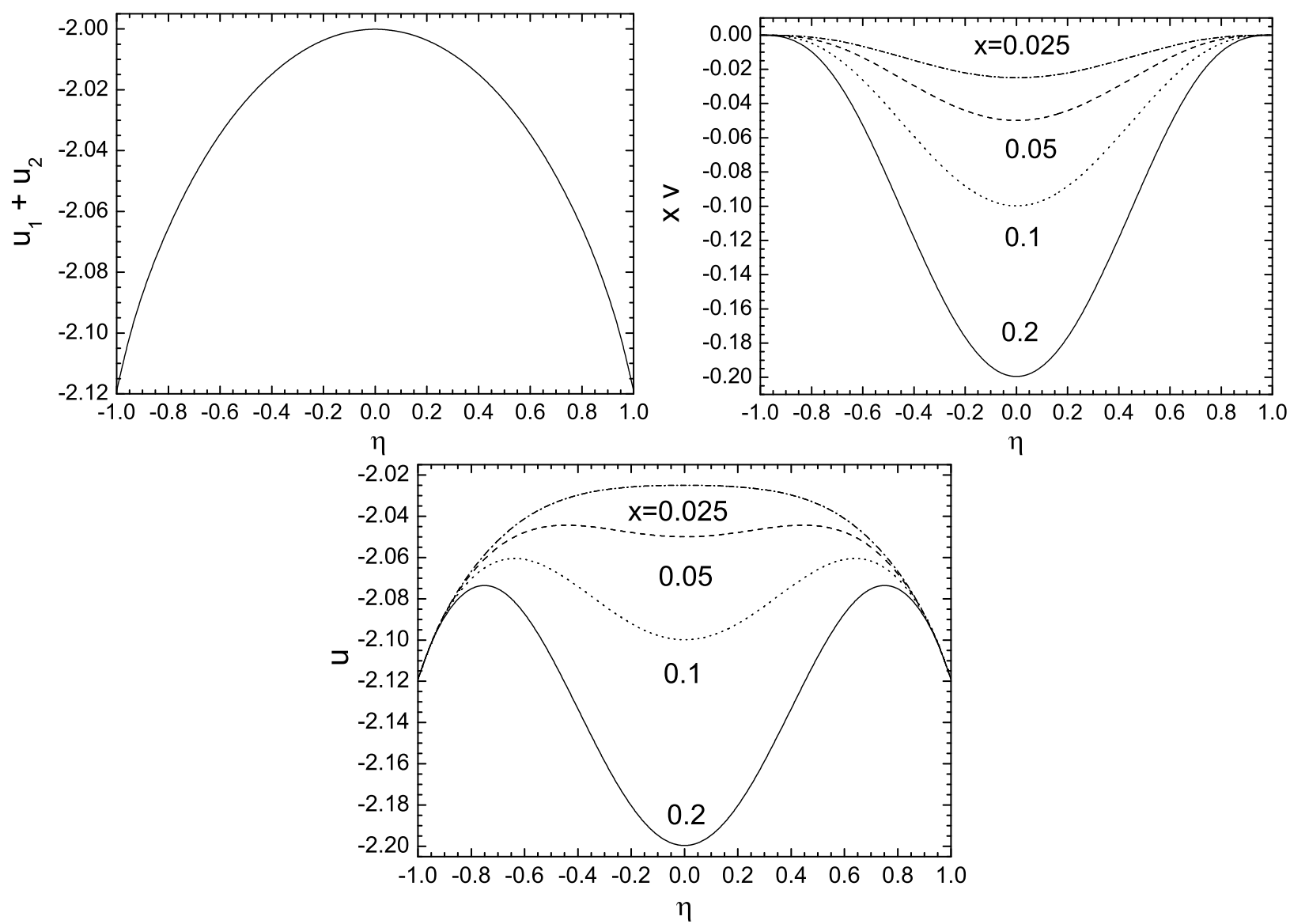

FIG. 2: The calculated dimensionless $u, u_{1}+u_{2}$, and $x v$ vs $\eta$ at indicated $x$. 

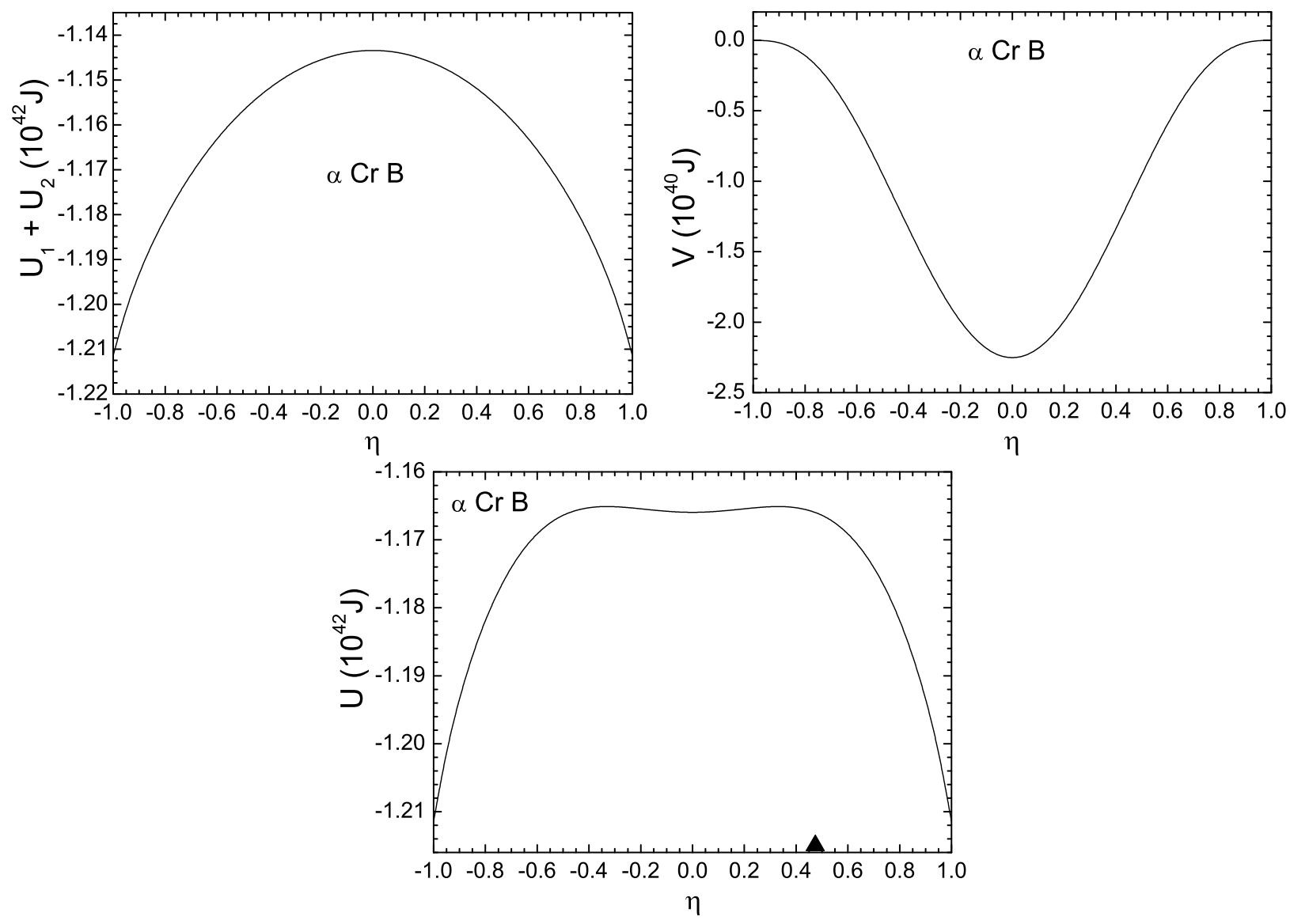

FIG. 3: The calculated gravitational energy $U_{1}+U_{2}$, star-star interaction energy, and total potential energy $U$ vs $\eta$ for close binary star $\alpha$ Cr B. The arrows on $x$-axis show the initial $\eta_{i}$. 

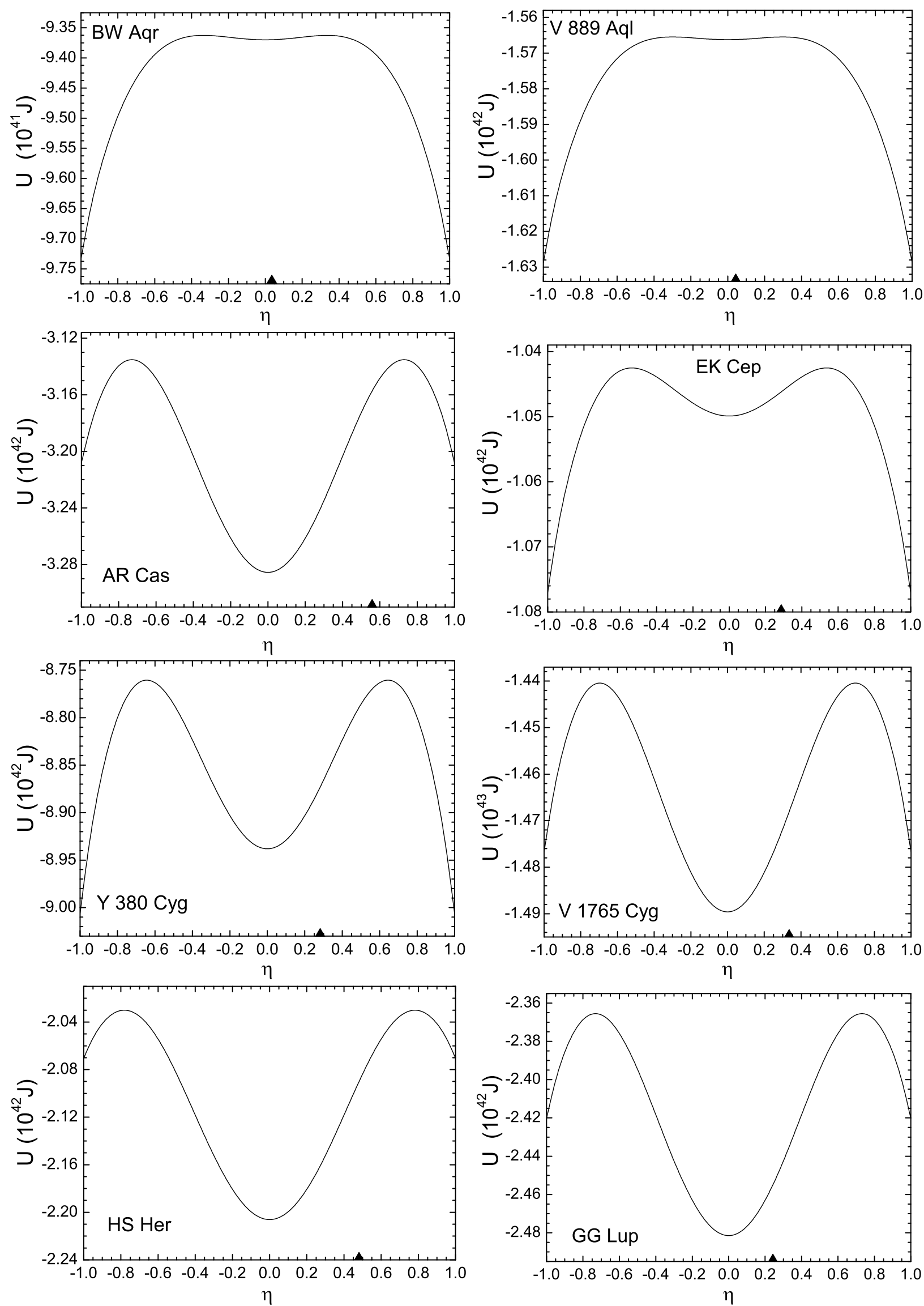

FIG. 4: The calculated total potential energie $5 U$ vs $\eta$ for the indicated close binary stars. The arrows on $x$-axis show the corresponding initial $\eta_{i}$ for binary stars. 

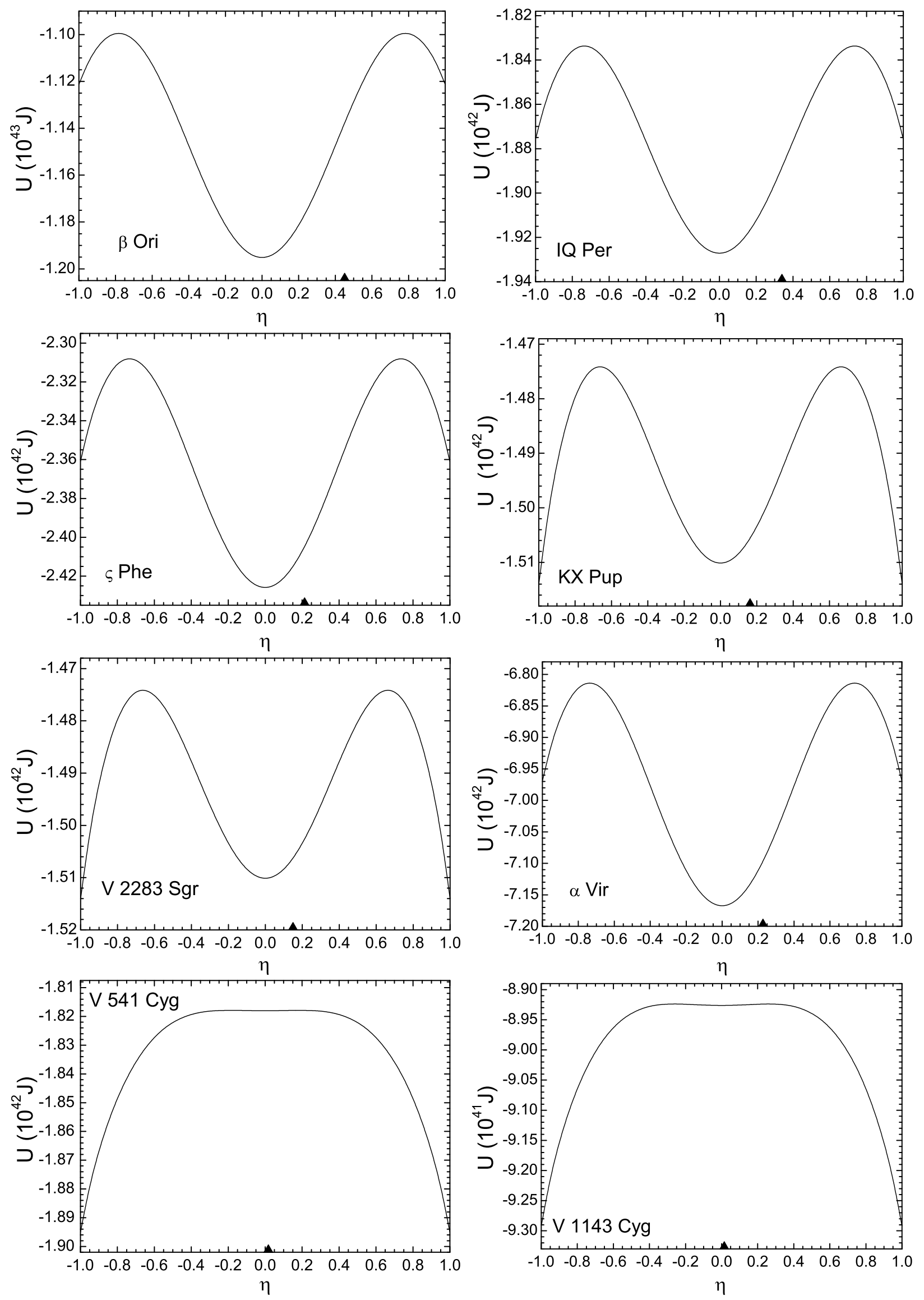

FIG. 5: The same as in Fig. 4, but16or other indicated close binary stars. 

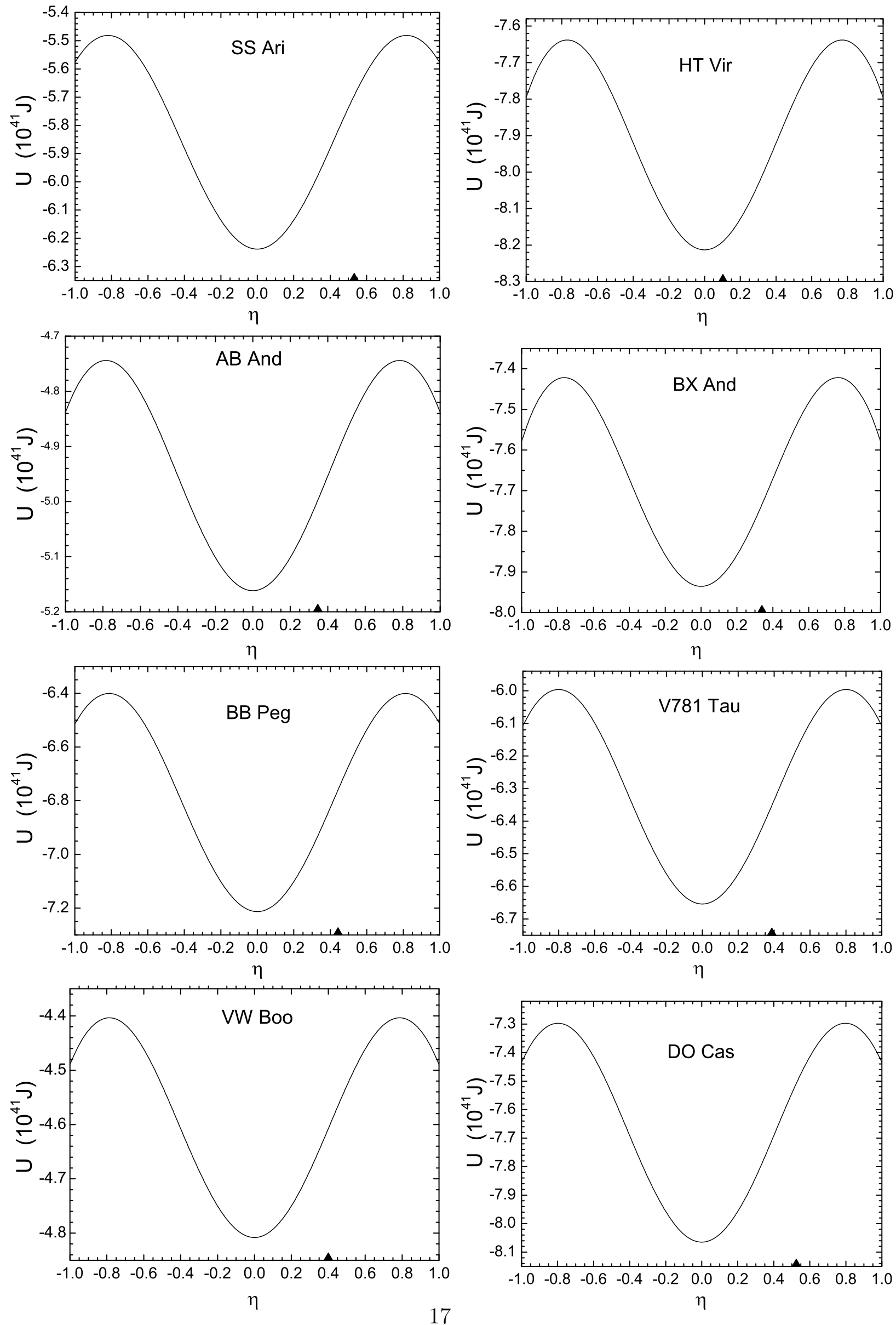

FIG. 6: The same as in Fig. 4, but for other indicated close binary stars. 

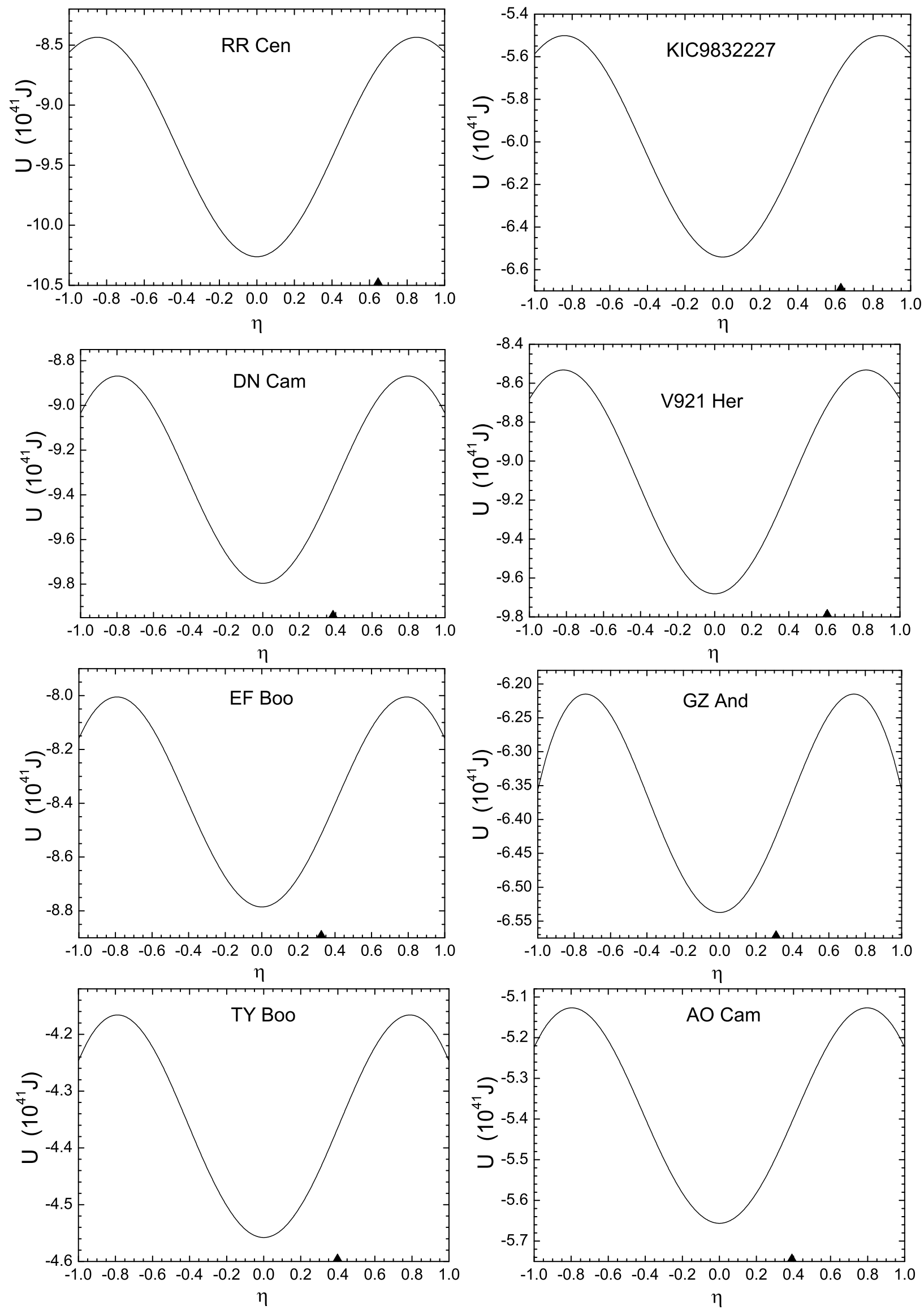

FIG. 7: The same as in Fig. 4, but1 for other indicated close binary stars. 
TABLE I: The calculated $\omega_{1}, \omega_{2}, \beta / \alpha, \Delta U=U\left(\eta_{i}\right)-U(\eta=0), B_{\eta}=U\left(\eta_{b}\right)-U\left(\eta_{i}\right)$, and observed data $M_{1} / M_{\odot}, M_{2} / M_{\odot}[6$, , 7] for the close binary stars indicated.

\begin{tabular}{|c|c|c|c|c|c|c|c|}
\hline Di-star & $\frac{M_{1}}{M_{\odot}}$ & $\frac{M_{2}}{M_{\odot}}$ & $\omega_{1}$ & $\omega_{2}$ & $\beta / \alpha$ & $\Delta U(\mathrm{~J})$ & $B_{\eta}(\mathrm{J})$ \\
\hline BW Aqr & 1.48 & 1.38 & 1.49 & 1.5 & 0.040 & $2 \times 10^{37}$ & $7 \times 10^{38}$ \\
\hline V 889 Aql & 2.4 & 2.2 & 1.32 & 1.35 & 0.038 & $3 \times 10^{37}$ & $8 \times 10^{38}$ \\
\hline V 539 Ara & 6.24 & 5.31 & 1.04 & 1.08 & 0.150 & $5 \times 10^{39}$ & $2 \times 10^{41}$ \\
\hline AS Cam & 3.31 & 2.51 & 1.22 & 1.31 & 0.098 & $4 \times 10^{39}$ & $3 \times 10^{40}$ \\
\hline EM Car & 22.8 & 21.4 & 0.75 & 0.76 & 0.309 & $8 \times 10^{39}$ & $2 \times 10^{42}$ \\
\hline GL Car & 13.5 & 13.0 & 0.86 & 0.87 & 0.287 & $10^{39}$ & $10^{42}$ \\
\hline QX Car & 9.27 & 8.48 & 0.94 & 0.96 & 0.152 & $2 \times 10^{39}$ & $3 \times 10^{41}$ \\
\hline AR Cas & 6.7 & 1.9 & 1.02 & 1.40 & 0.170 & $10^{41}$ & $2 \times 10^{40}$ \\
\hline IT Cas & 1.40 & 1.40 & 1.51 & 1.51 & 0.056 & 0 & $4 \times 10^{39}$ \\
\hline OX Cas & 7.20 & 6.30 & 1 & 1.04 & 0.192 & $5 \times 10^{39}$ & $3 \times 10^{41}$ \\
\hline PV Cas & 2.79 & 2.79 & 1.27 & 1.27 & 0.144 & 0 & $7 \times 10^{40}$ \\
\hline KT Cen & 5.30 & 5.00 & 1.08 & 1.27 & 0.116 & $4 \times 10^{38}$ & $10^{41}$ \\
\hline V 346 Cen & 11.8 & 8.4 & 0.89 & 0.97 & 0.137 & $3 \times 10^{40}$ & $2 \times 10^{41}$ \\
\hline CW Cep & 11.60 & 11.10 & 0.89 & 0.9 & 0.243 & $10^{39}$ & $7 \times 10^{41}$ \\
\hline EK Cep & 2.02 & 1.12 & 1.38 & 1.6 & 0.066 & $4 \times 10^{39}$ & $4 \times 10^{39}$ \\
\hline Y Cyg & 17.5 & 17.3 & 0.8 & 0.81 & 0.292 & $2 \times 10^{38}$ & $2 \times 10^{41}$ \\
\hline Y 380 Cyg & 14.3 & 8.0 & 0.85 & 1.27 & 0.103 & $7 \times 10^{40}$ & $10^{41}$ \\
\hline V 453 Cyg & 14.5 & 11.3 & 0.84 & 0.9 & 0.213 & $4 \times 10^{40}$ & $7 \times 10^{41}$ \\
\hline V 477 Cyg & 1.79 & 1.35 & 1.42 & 1.53 & 0.088 & $2 \times 10^{39}$ & $10^{40}$ \\
\hline V 478 Cyg & 16.6 & 16.3 & 0.81 & 0.82 & 0.290 & $4 \times 10^{38}$ & $10^{42}$ \\
\hline V 541 Cyg & 2.69 & 2.60 & 1.28 & 1.29 & 0.033 & $2 \times 10^{36}$ & $10^{38}$ \\
\hline V 1143 Cyg & 1.39 & 1.35 & 1.51 & 1.53 & 0.036 & $2 \times 10^{36}$ & $2 \times 10^{38}$ \\
\hline V 1765 Cyg & 23.5 & 11.7 & 0.75 & 0.89 & 0.138 & $2 \times 10^{41}$ & $3 \times 10^{41}$ \\
\hline CO Lac & 3.13 & 2.75 & 1.24 & 1.28 & 0.163 & $2 \times 10^{39}$ & $9 \times 10^{40}$ \\
\hline
\end{tabular}


TABLE II: The same as in Table I, but for other close binary stars.

\begin{tabular}{|c|c|c|c|c|c|c|c|}
\hline Di-star & $\frac{M_{1}}{M_{\odot}}$ & $\frac{M_{2}}{M_{\odot}}$ & $\omega_{1}$ & $\omega_{2}$ & $\beta / \alpha$ & $\Delta U(\mathrm{~J})$ & $B_{\eta}(\mathrm{J})$ \\
\hline DI Her & 5.15 & 4.52 & 1.09 & 1.13 & 0.060 & $7 \times 10^{38}$ & $2 \times 10^{40}$ \\
\hline HS Her & 4.25 & 1.49 & 0.89 & 1.49 & 0.258 & $10^{41}$ & $10^{41}$ \\
\hline GG Lup & 4.12 & 2.51 & 1.15 & 1.3 & 0.173 & $3 \times 10^{40}$ & $10^{41}$ \\
\hline RU Mon & 3.60 & 3.33 & 1.19 & 1.22 & 0.101 & $4 \times 10^{38}$ & $5 \times 10^{40}$ \\
\hline GN Nor & 2.50 & 2.50 & 1.31 & 1.31 & 0.061 & 0 & $10^{40}$ \\
\hline U Oph & 5.02 & 4.52 & 1.1 & 1.13 & 0.203 & $2 \times 10^{39}$ & $2 \times 10^{41}$ \\
\hline V $451 \mathrm{Oph}$ & 2.77 & 2.35 & 1.27 & 1.33 & 0.119 & $2 \times 10^{39}$ & $5 \times 10^{40}$ \\
\hline$\beta$ Ori & 19.8 & 7.5 & 0.87 & 0.99 & 0.259 & $6 \times 10^{41}$ & $4 \times 10^{41}$ \\
\hline FT Ori & 2.50 & 2.30 & 1.31 & 1.33 & 0.089 & $3 \times 10^{38}$ & $5 \times 10^{40}$ \\
\hline AG Per & 5.36 & 4.90 & 1.08 & 1.1 & 0.187 & $2 \times 10^{39}$ & $2 \times 10^{41}$ \\
\hline IQ Per & 3.51 & 1.73 & 1.2 & 1.43 & 0.177 & $4 \times 10^{40}$ & $6 \times 10^{40}$ \\
\hline$\varsigma$ Phe & 3.93 & 2.55 & 1.17 & 1.3 & 0.178 & $2 \times 10^{40}$ & $10^{41}$ \\
\hline KX Pup & 2.5 & 1.8 & 1.31 & 1.42 & 0.114 & $5 \times 10^{39}$ & $3 \times 10^{40}$ \\
\hline NO Pup & 2.88 & 1.5 & 1.26 & 1.49 & 0.192 & $3 \times 10^{40}$ & $6 \times 10^{40}$ \\
\hline VV Pyx & 2.10 & 2.10 & 1.37 & 1.37 & 0.064 & 0 & $10^{40}$ \\
\hline YY Sgr & 2.36 & 2.29 & 1.33 & 1.33 & 0.099 & $4 \times 10^{37}$ & $3 \times 10^{40}$ \\
\hline V 523 Sgr & 2.1 & 1.9 & 1.37 & 1.4 & 0.098 & $3 \times 10^{38}$ & $3 \times 10^{40}$ \\
\hline V 526 Sgr & 2.11 & 1.66 & 1.36 & 1.45 & 0.111 & $2 \times 10^{39}$ & $3 \times 10^{40}$ \\
\hline V 1647 Sgr & 2.19 & 1.97 & 1.35 & 1.39 & 0.080 & $3 \times 10^{38}$ & $2 \times 10^{40}$ \\
\hline V $2283 \mathrm{Sgr}$ & 3.0 & 2.22 & 1.25 & 1.25 & 0.092 & $4 \times 10^{39}$ & $3 \times 10^{40}$ \\
\hline V 760 Sco & 4.98 & 4.62 & 1.1 & 1.12 & 0.199 & $10^{39}$ & $2 \times 10^{41}$ \\
\hline AO Vel & 3.2 & 2.9 & 1.23 & 1.26 & 0.163 & $10^{39}$ & $10^{41}$ \\
\hline EO Vel & 3.21 & 2.77 & 1.23 & 1.27 & 0.072 & $7 \times 10^{38}$ & $2 \times 10^{40}$ \\
\hline$\alpha \mathrm{Vir}$ & 10.8 & 6.8 & 0.91 & 0.99 & 0.180 & $7 \times 10^{40}$ & $3 \times 10^{41}$ \\
\hline DR Vul & 13.2 & 12.1 & 1.03 & 0.88 & 0.295 & $7 \times 10^{39}$ & $10^{42}$ \\
\hline
\end{tabular}


TABLE III: The same as in Table I, but for contact binary stars. The observed data $M_{1} / M_{\odot}$, $M_{2} / M_{\odot}$ are from Refs. [12, 13].

\begin{tabular}{|c|c|c|c|r|r|r|r|}
\hline Di-star & $\frac{M_{1}}{M_{\odot}}$ & $\frac{M_{2}}{M_{\odot}}$ & $\omega_{1}$ & $\omega_{2}$ & $\beta / \alpha$ & $\Delta U(\mathrm{~J})$ & $B_{\eta}(\mathrm{J})$ \\
\hline AB And & 1.01 & 0.49 & 1.64 & 1.96 & 0.261 & $2 \times 10^{40}$ & $3 \times 10^{40}$ \\
GZ And & 1.12 & 0.59 & 1.60 & 1.88 & 0.283 & $2 \times 10^{40}$ & $4 \times 10^{40}$ \\
OO Aql & 1.05 & 0.88 & 1.62 & 1.70 & 0.180 & $10^{39}$ & $3 \times 10^{40}$ \\
V417 Aql & 1.40 & 0.50 & 1.51 & 1.96 & 0.359 & $5 \times 10^{40}$ & $3 \times 10^{40}$ \\
SS Ari & 1.31 & 0.40 & 1.54 & 2.07 & 0.372 & $6 \times 10^{40}$ & $2 \times 10^{40}$ \\
V402 Aur & 1.64 & 0.33 & 1.45 & 2.17 & 0.512 & $10^{41}$ & $10^{40}$ \\
TY Boo & 0.93 & 0.40 & 1.67 & 2.07 & 0.275 & $2 \times 10^{40}$ & $2 \times 10^{40}$ \\
EF Boo & 1.61 & 0.82 & 1.46 & 1.73 & 0.282 & $3 \times 10^{40}$ & $5 \times 10^{40}$ \\
AO Cam & 1.12 & 0.49 & 1.60 & 1.97 & 0.295 & $3 \times 10^{40}$ & $3 \times 10^{40}$ \\
DN Cam & 1.85 & 0.82 & 1.41 & 1.73 & 0.298 & $4 \times 10^{40}$ & $5 \times 10^{40}$ \\
TX Cnc & 0.91 & 0.50 & 1.68 & 1.96 & 0.212 & $9 \times 10^{39}$ & $2 \times 10^{40}$ \\
RR Cen & 2.09 & 0.45 & 1.37 & 2.01 & 0.542 & $2 \times 10^{41}$ & $2 \times 10^{40}$ \\
V752 Cen & 1.30 & 0.40 & 1.54 & 2.07 & 0.391 & $6 \times 10^{40}$ & $2 \times 10^{40}$ \\
V757 Cen & 0.88 & 0.59 & 1.70 & 1.88 & 0.212 & $5 \times 10^{39}$ & $3 \times 10^{40}$ \\
VW Cep & 0.93 & 0.40 & 1.67 & 2.07 & 0.300 & $2 \times 10^{40}$ & $2 \times 10^{40}$ \\
TW Cet & 1.06 & 0.61 & 1.62 & 1.86 & 0.258 & $10^{40}$ & $4 \times 10^{40}$ \\
RW Com & 0.56 & 0.20 & 1.90 & 2.46 & 0.283 & $10^{40}$ & $8 \times 10^{39}$ \\
RZ Com & 1.23 & 0.55 & 1.56 & 1.91 & 0.303 & $3 \times 10^{40}$ & $3 \times 10^{40}$ \\
V921 Her & 2.07 & 0.51 & 1.37 & 1.95 & 0.364 & $10^{41}$ & $2 \times 10^{40}$ \\
\hline
\end{tabular}

